\title{
Applications of nanoparticels in molecular and cellular biology and cancer research
}

\author{
Katya Simeonova ${ }^{1 *}$, Ganka Milanova $^{2}$ \\ From 22nd European Society for Animal Cell Technology (ESACT) Meeting on Cell Based Technologies \\ Vienna, Austria. 15-18 May 2011
}

\begin{abstract}
Background
Discovering of carbon nanotubes (CNTs), by S. Iijima in 1991, [1] was a revolution in nanoscience (nanomaterials) and nanotechnology. Moreover these nanoscale materials possess perfectly physico-mechanicanical, electronic, optical, properties. They find applications in technique, engineering, electronics, optoelectronis, space and environments, [2]. Recently has been established that nanomaterials, play an important role in molecular and cellular biology and medicine. The aim of the work presented could be formulated as follows: to discuss some basic articles, devoted to applications of nanoparticles, nanotechnology based on gold nanoparticles for cancer research.
\end{abstract}

\section{Materials and methods}

Synthesis methods for nanoparticles (nanoshells, nanorods, nanocrystals) have been analyzed. Application of gold nanoparticels for detection and therapy of cancer has been given too, [3]. Nanotechnology has been determined as an interdisciplinary science combining physics, mechanics, chemistry, materials science, engineering, biology becames a very good potential in many different fields of technique, for cancer therapy, in molecular and cellular biology.

\section{Methods for synthesis of nanoparticles}

Some methods for synthesis of nanoparticles: by controlled different reducing agent; a two- phase method using other reductants; biocompatible bock polymers. Deposition process (DP), has been applied for synthesis also. It has been established that rods wires, multi-concentric shells, hollow tubes, capsules, monocrystas etc. possess exceptional optical and electronic properties. In

\footnotetext{
* Correspondence: katyas@bas.bg

${ }^{1}$ Institute of Mechanics, Bulgarian Academy of Sciences, 1113 Sofia, Bulgaria Full list of author information is available at the end of the article
}

[4], the Drude method (model) for description of optical properties is:

$$
\begin{aligned}
& \varepsilon^{\prime}=1-\frac{\omega_{p}{ }^{2}}{\omega^{2}+\gamma^{2}} \\
& \varepsilon^{\prime \prime}=1-\frac{\omega_{p}{ }^{2} \gamma}{\omega\left(\omega^{2}+\gamma^{2}\right)}
\end{aligned}
$$

Here: $\varepsilon^{\prime}$ and $\varepsilon^{\prime \prime} ; \omega=2 \pi c / \lambda ; \lambda, c, \gamma$ have been given in [5]. A correlation, available is:

$$
\gamma=\gamma_{\text {bulk }}+\frac{v_{F}}{r_{\text {eff }}}
$$

Here $\gamma, \gamma_{\text {bulk }}, v_{F}, r_{\text {off }}$ are given in [5]. Both important characteristics for description of gold nanoparticles, absorption efficiency and scattering efficiency have been analyzed too. It must be pointed out as well, that these nanoparticles could employed in the many medical applications.

\section{Results}

The paper presented could be considered as a recent review on application of nanoparticles and nanotechnology in cancer research. In the work [6], we could find basic research of American scientists in nanotechnology based for cancer research, (Figure 1).

\section{Conclusions}

In conclusions we could say, that paper presented could be a successful tool for many medical scientists, physicians, molecular biology scientists, chemists etc. It's give good knowledge, regarding nanotechnology based gold nanoparticles for cancer research. Also, some novel 


\section{Nanoshells: Photothermal therapy}

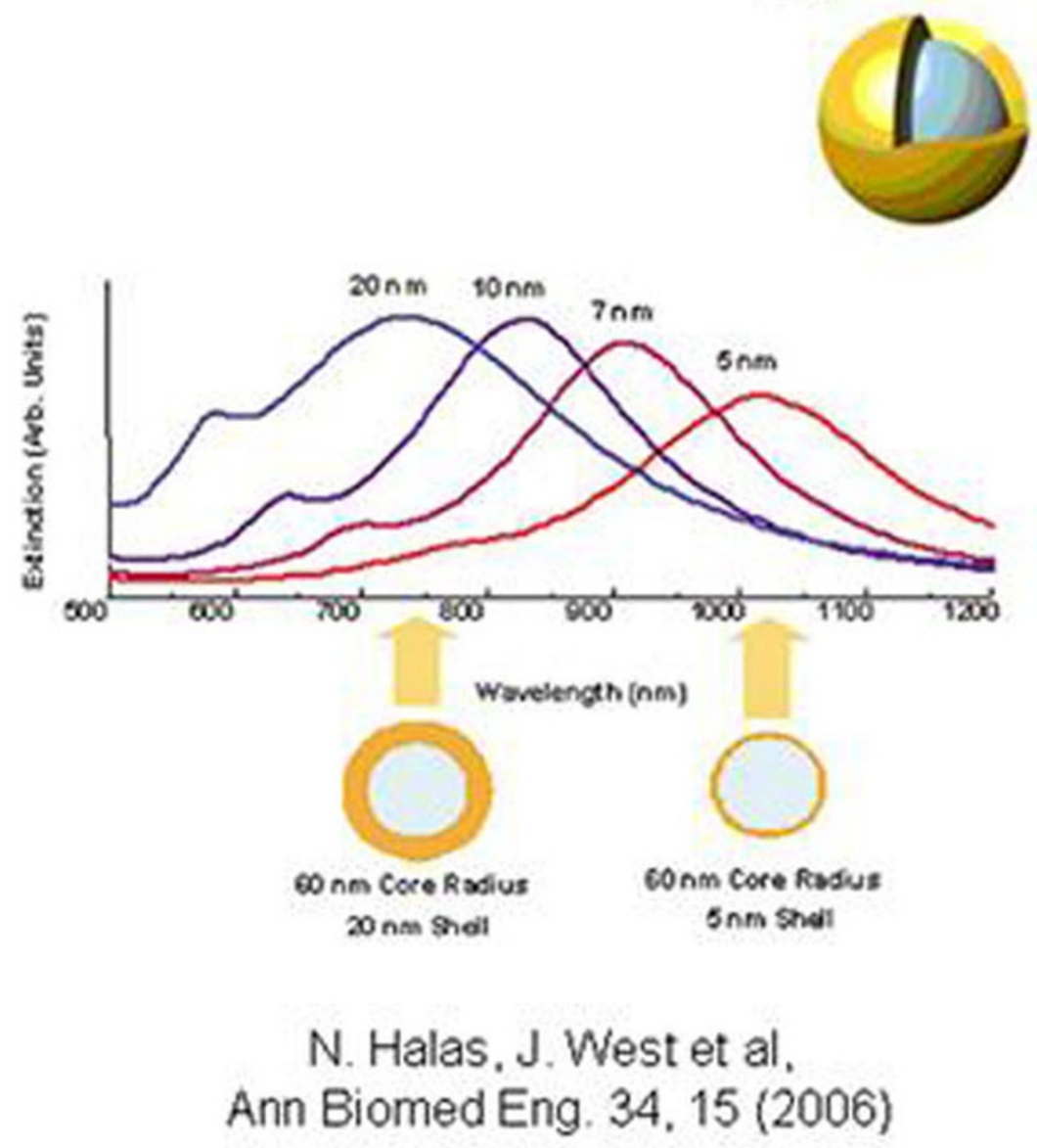

Figure 1 Dependencies of extinction, versus wavelength for nanoshells with different core radius

computational models, based on theoretical studies, analyzed here, could be developed in future inestigations.

\section{Acknowledgments}

Authors would like to thank you very much to Professor Nicole Borth, personally for her cooperation of attending the ESACT Meeting, in Vienna, Austria from 15-18 May 2011

\section{Author details}

${ }^{1}$ Institute of Mechanics, Bulgarian Academy of Sciences, 1113 Sofia, Bulgaria. 2University of Architecture, Civil Engineering and Geodesy, 1000 Sofia, Bulgaria.
3. Weibo C, et al: Applications of gold nanoparticles in cancer nanotechnology. Review, Nanotechnology, Science and Applications 2008, 27-32.

4. Lihua W, et al: Gold nanoparticle- based optical probes for targetresponsive DNA structures. Gold Bulletin 2008, 41(1):37-42.

5. Harris $N$, et al: Tunable integrated absorption by metal nanoparticles: the case for gold rods and shells. Gold Bulletin 2008, 41(1):5-14.

6. NCl Alliance for Nanotechnology in Cancer. nanoUtah, Piotr Grodzinski, PhD, Director 2007.

doi:10.1186/1753-6561-5-S8-P58

Cite this article as: Simeonova and Milanova: Applications of nanoparticels in molecular and cellular biology and cancer research. BMC Proceedings 2011 5(Suppl 8):P58.

Published: 22 November 2011

\section{References}

1. lijima S: Nature. 1991, 395.

2. Simeonova K, Milanova G: A review on the mechanical behavior of carbon nanotubes (CNTs), as semiconductors. ISCOM2007, Book of abstracts Peniscola, Spain; 2007, 123-136. 